\title{
Plasma Physics and Controlled Nuclear Fusion Research
}

From 6 to 13 October, 1976, the 6th International Conference on Plasma Physics and Controlled Nuclear Fusion Research, organized by the International Atomic Energy Agency, was held in Berchtesgaden in the Bavarian mountains. The number of participants, a large part of whom (about $60 \%$ ) came from the EPS area, was as usual at this series of conferences, of the order 500 .

The large majority of the papers read at the conference were concerned with magnetic plasma confinement, the field which continues to receive the strongest interest in the framework of fusion research, and within which work on tokamaks retains the first place. Two tokamaks of increased size, T-10 at the Kurchatov Institute in Moscow and PLT Princeton, have come into operation during the past year, and first result were presented at the conference. Although it is definitely too early to draw from them, conclusions about the scaling of tokamak confinement, it can be said that the increase in the minor radius of the torus has not led to major problems. The expected increase in confinement time has been achieved, and, again as expected, the electron temperatures reached by Ohmic heating are in the same range (ca. $1 \mathrm{keV}$ ) as in smaller machines. Furthermore, in the device T-10 the plasma is found to contain less impurities, possibly due to a reduction of wall bombardment by highly energetic neutrals as a consequence of the larger plasma size. An analogous effect has also been found when smaller experiments (Alcator at MIT and Pulsator at Garching) are operated at high densities. A further important result of these latter experiments is that the plasma confinement improves (about linearly) with plasma density. Values of $10^{13} \mathrm{~cm}^{-3}$ for the product, plasma density times energy confinement time, have been reached in Alcator. Additional heating by injection of energetic neutrals has been raised to the $0.5 \mathrm{MW}$ level in TFR at Fontenay-aux-Roses and, somewhat below, in ORMAK at Oak Ridge and DITE at Culham, yielding an increase of the ion temperature by a factor two to three. This is consistent with collisional transport being responsible for the heat conduction of the ions. Preliminary results from DITE show the bundle divertor to be effective for controlling plasmawall interactions. Moreover, many new results have been reported which increasingly improve the understanding of the complex physical phenomena appearing in tokamak devices.

Stellerator experiments have also been scaled up in size, but they have only just reached the dimension of the tokamaks which became operational almost a decade earlier. The three new large devices are CLEO in Culham, L-2 at the Lebedev Institute at Moscow, and W VII A in Garching. There are indications that in the absence of Ohmic heating currents, the stability, and consequently confinement, properties of a Stellerator improve above those of a comparable tokamak. Analogous results have also been found in the Culham Levitron.

Stable screw-pinch discharges of $0.1 \mathrm{~ms}$ duration and reaching values of the ratio $\beta$ of kinetic and magnetic pressure of 0.2 have been generated in the SPICA experiment in Jutphaas. Progress has also been made with the theoretical analysis of the physics of high- $\beta$ discharges.

The effectiveness of improving the confinement properties of mirror systems by providing a stream of plasma along the magnetic field to stabilize the loss-cone instability has been further assessed in the II X-2 device at Livermore. In a very interesting proposal, put forward by G.U. Dimov et al. from Novosibirsk, and independently made by the Livermore group, it was suggested that advantage be taken of electrostatic confinement in mirrors by putting three mirrors in series, the two outer ones being much shorter than the main mirror in the middle and filled with a very hot and dense plasma.

Regarding inertial confinement, pellet volume compression ratios, by irradiation of laser light, up to 2000 were reported by the group at KMS (Ann Arbor). Much work is done to analyse the very involved physical phenomena appearing when an intense laser beam interacts with matter. First encouraging results on pellet compression by relativistic electron beams were presented by a group from the Kurchatov Institute. From the neutron yield found, in fact, a compression ratio of 1000 may be inferred, showing that the method is competitive with compression by laser beams.

\section{F. Engelmann}

\section{EPS Divisions}

An election will take place early in 1977 for membership of the Nuclear Physics Board. Board members serve for a period of 3 years with the possibility of re-election for a further 3 year period. The following members of the Board are willing to serve for a further term : K. Bethge, N. Cindro, E. Cotton, M. Ivascu, R. Ricci, V. SoIoviev, A. Strzalkowski, I. Talmi and C. Van der Leun. Not offering themselves for re-election are: K. Adler, W. D. Hamilton, S. Nilsson and T. Mayer-Kuckuk. It is hoped that other nuclear physicists will be willing to stand, but the Board is an active one and we are anxious that anyone who is elected will be prepared to take a full part both at the Board meetings and in the activities of the Division. Board meetings are usually held twice a year, in conjunction (when possible) with Divisional conferences but it should be added that no funds are available for travel or subsistence. Nominations should be supported by the signatures of 4 Individual Ordinary Members of the EPS and should be sent to the Executive Secretary, EPS Secretariat.

W. D. H.

The new chairman of the Plasma Physics Division is $\mathrm{J}$. $\mathrm{L}$. Delcroix of the Laboratoire de Physique des Plasmas, Orsay.

The new chairman of the Quantum Electronics Division is F. K. Kneubühl of ETH, Zurich.

The Executive Committee, on the recommendation of the Programme Committee, has decided to give the Fourth General Conference of EPS to be held in York, 25-29 September, 1978, the title of Trends in Physics, re-establishing the title of the Second General Conference held in Wiesbaden in 1972.

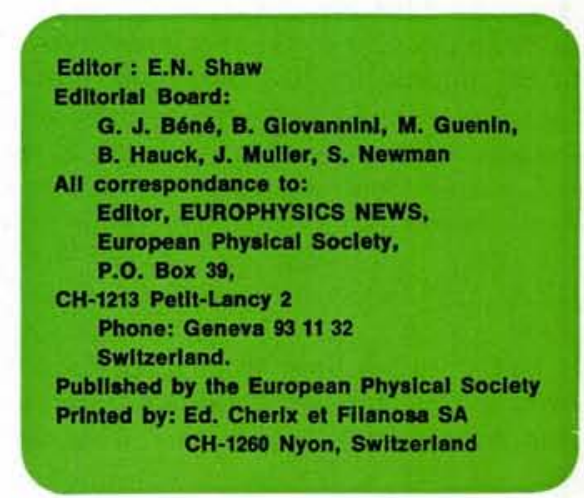

